\title{
Omicron Leonis, an evolving Am binary: when two wrongs do make a right
}

\author{
R. Elizabeth M. Griffin \\ Herzberg Institute for Astrophysics, DAO, Canada
}

\begin{abstract}
Both components of the composite-spectrum binary o Leo have Am characteristics, even though the primary is an evolving giant $(\log g=3.25)$ with $T_{\text {eff }} \sim 6100 \mathrm{~K}$. This is believed to be the first isolation of such a cool Am star. The finding challenges the theories of diffusion which are widely accepted as the cause of metallicism. The primary component ( $o$ Leo A) appears to be deficient in $\mathrm{Ca}$ and $\mathrm{Sc}$, as are classical Am stars. Its unusual state may be attributable either to its current state of rapid evolution, or to regular Am-star evolution that is difficult to recognize spectroscopically. A full account of this research appeared in AJ 123, 988-1001, 2002.
\end{abstract}

Keywords. Stars: binaries: spectroscopic, stars: chemically peculiar, stars: individual: (o Leonis), stars: evolution, techniques: spectroscopic

\section{Introduction}

The component spectra of over 30 composite-spectrum binaries (to date) have been isolated successfully by the spectrum-subtraction method. For the great majority it has been possible to measure a precise mass ratio (simply as the reciprocal of the radialvelocity ratio at any point in the orbit). o Leo $(V=3.5)$ is a good candidate for such analysis. It is clearly double-lined (see Fig. 2), and both components have fairly narrow lines.

Both stars give a well-defined 'dip' with a Coravel RV instrument, so in this case the mass ratio was determined reliably from the double-lined orbit (Fig. 1, the orbit was determined by R. F. Griffin). The period is 14.5 days.

There is a good-quality astrometric orbit for $o$ Leo as well as a precise distance, so $M_{V}$, $\Delta M_{V}, \sin i$ and the individual masses were already known. But we wanted to know what kind of sharp-lined cool giant could exist in an orbit of only 14.5 days.

\section{Spectrum subtraction}

We attempted to determine the individual spectral types by isolating the spectra. All we had to do in principle was to find which standard spectrum best matched that of $o$ Leo A, and subtract the right fraction of it so as to uncover the secondary spectrum.

That was easier said than done. We could not match the primary! It appeared to be near G0, but its lines were substantially deeper than those of any normal G-type giant. Instead of uncovering a single spectrum of a recognizeable type, the trial subtractions resulted in something more complex than the original.

\section{High-resolution spectra}

\subsection{Photographic spectra}

The routine dispersion is $10 \AA \mathrm{mm}^{-1}$, so next we observed the composite spectrum at $6.5 \AA \mathrm{mm}^{-1}(\lambda 5600-6600 \AA)$ and $2.8 \AA \mathrm{mm}^{-1}(\lambda 3700-5000)$. Although we could only 


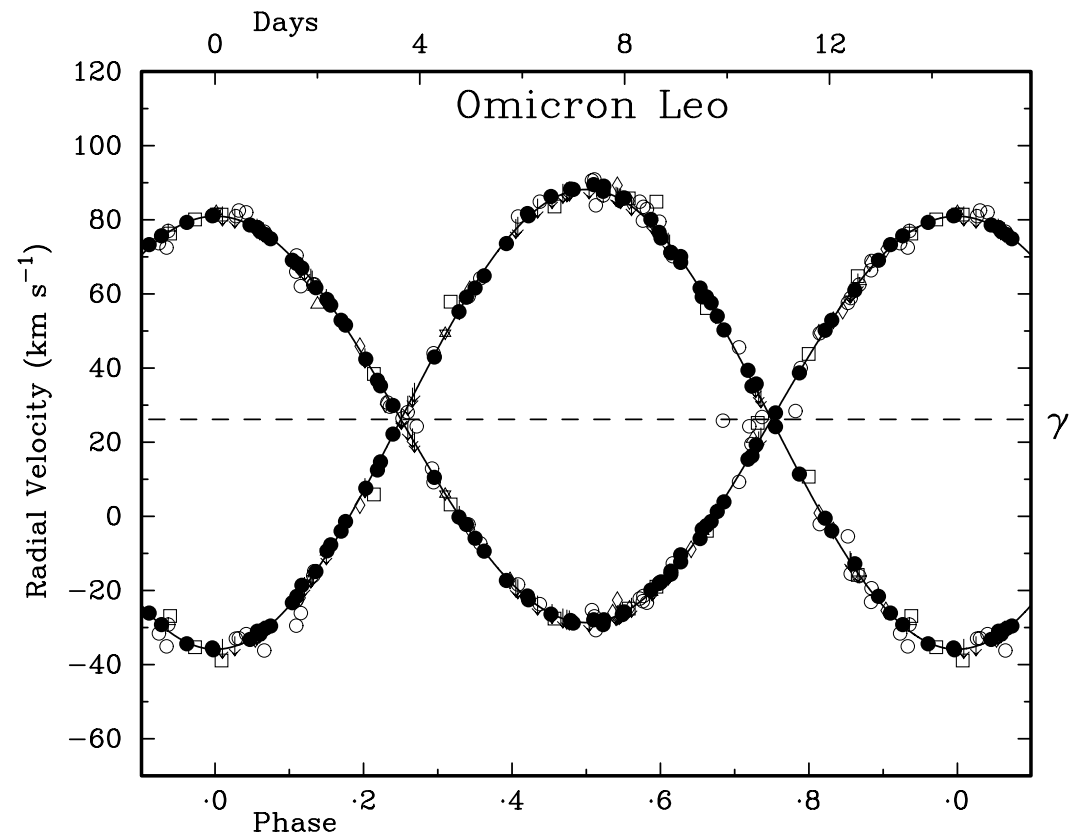

Figure 1. Double-lined orbit for $o$ Leo (kindly supplied by R. F. Griffin). The observations span $>100$ years,

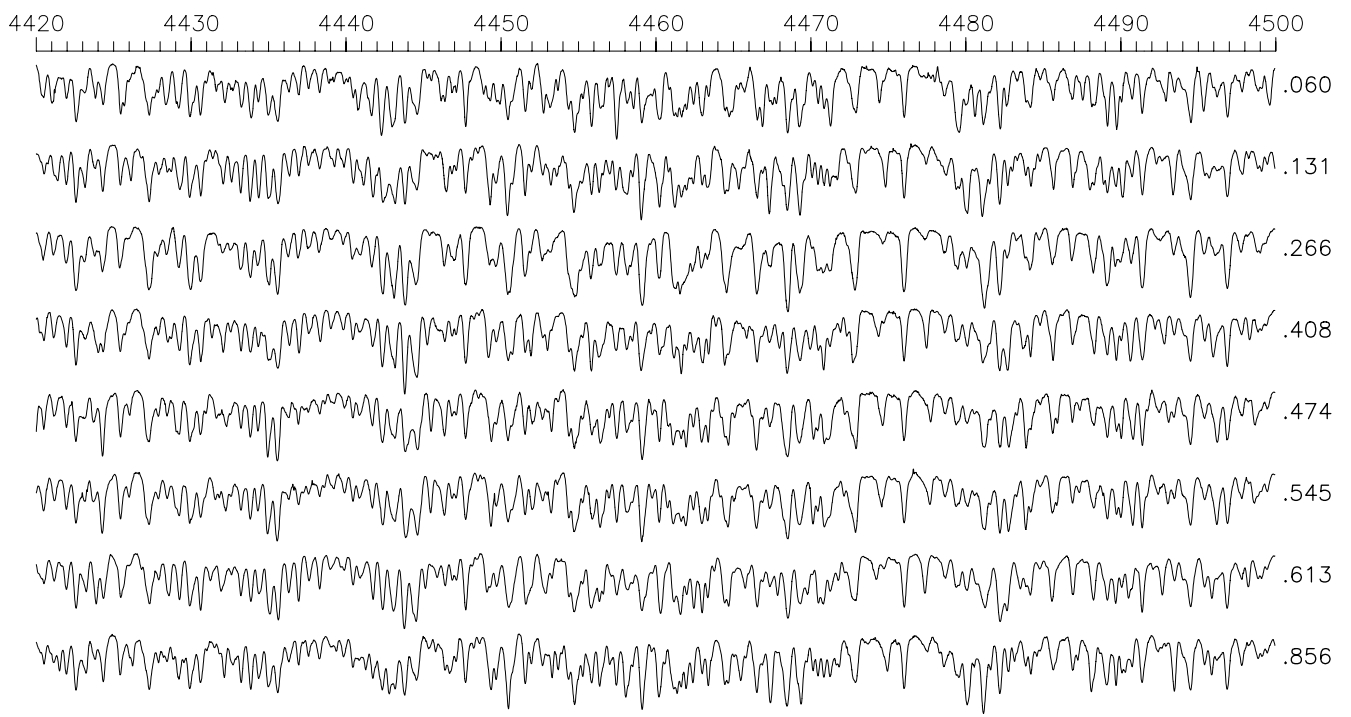

Figure 2. High-resolution spectra of the composite-spectrum binary o Leo at 8 different phases.

obtain 3 spectra at each wavelength, we became certain that the primary has an abnormal composition, with element enhancements similar to an Am or Ba type. The secondary, too, had to be metallic-lined because a normal A-type dwarf will not register a 'dip' with a Coravel RV spectrometer unless it has abnormally strong lines. But that knowledge did not help us to separate the spectra by our subtraction technique. 


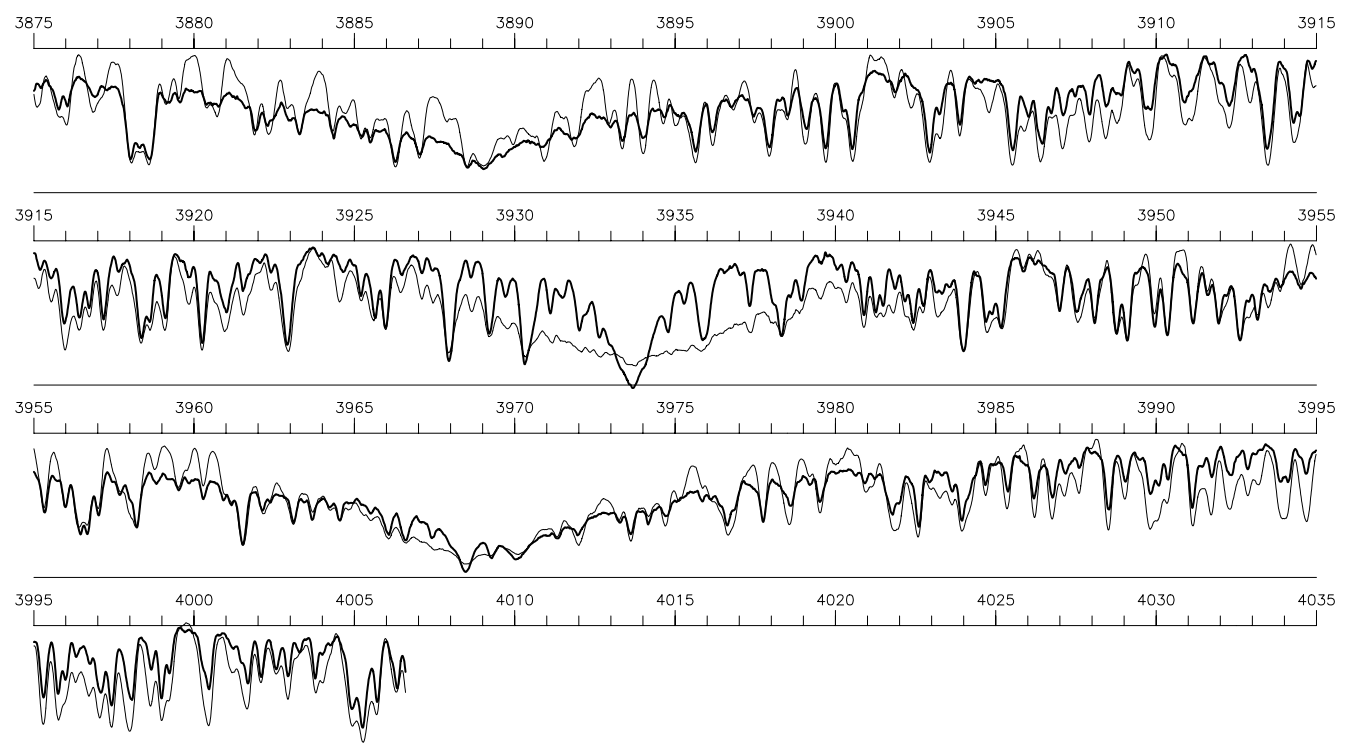

Figure 3. Disentangled and fully-restored spectra of $o$ Leo A (thin line) and $o$ Leo B (thick line) in the near UV.

\subsection{CCD spectroscopy}

We then obtained 26 high $S / N$, high-resolution spectra of $o$ Leo with the DAO $1.2-\mathrm{m}$ telescope. Half were centred near the Ca II K line at $\lambda 3933$ and half near Mg II $\lambda 4481$. Both sets included all phases of the orbit.

Again, it was possible to affirm that the $\mathrm{K}$ line in the giant was narrower than that in a giant near G0, and also that Ba II $\lambda 4554$ was somewhat enhanced in both components. But we still had no suitable standard to separate the spectra by subtraction. When spectra are abnormal it can be somewhat dangerous to proceed by spectrum subtraction, because a mismatch between (whichever) component creates spurious lines in the uncovered spectrum, and that can severely confuse its spectral classification. The component spectra are also rather similar to one another, which complicated the issue considerably.

\section{Unravelling the spectra}

We eventually separated the spectra successfully by applying the disentangling method FDBINARY, written as a package by S. Ilijić (see, Ilijić et al. 2001) from software originally developed by Hadrava (1995). The disentangling method separates the co-moving features in Fourier space, and averages them, so the more spectra one starts with, the higher the final S/N. FDBINARY was optimized for hot-star binaries (and preferably eclipsing ones), and applying it to a noneclipsing binary as cool as o Leo presented some initial problems.

\subsection{Classifying the component spectra}

Both spectra are abnormal. The secondary ( o Leo B) is mid- to late-Am. The classification of kA3hA7mF2 which we gave it places it in the category of 'classical' Am stars. Various pointers indicated a temperature of $7600 \pm 200 \mathrm{~K}$ and a radius of $2.6 R_{\odot}$.

The primary also shows all the characteristics of a classical Am star, yet its temperature was found to be $6100 \pm 200 \mathrm{~K}$, and its radius $5.7 R_{\odot}$. 
Vym

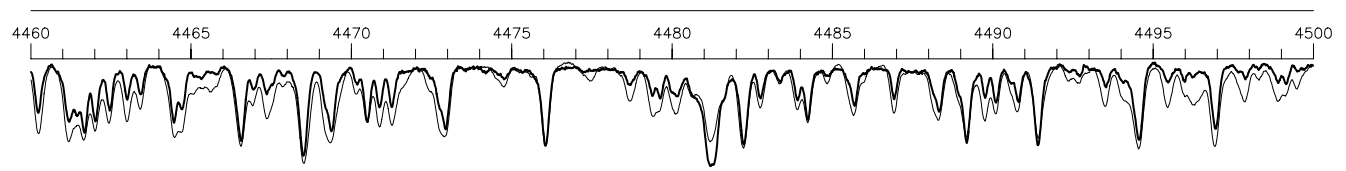

(5500

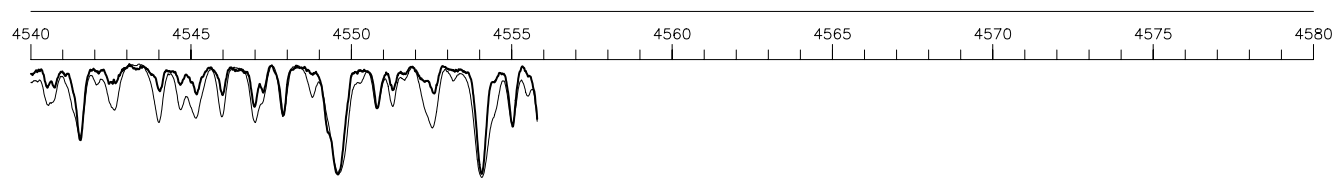

Figure 4. Disentangled and fully-restored spectra of $o$ Leo A (thin line) and $o$ Leo B (thick line) in the blue.

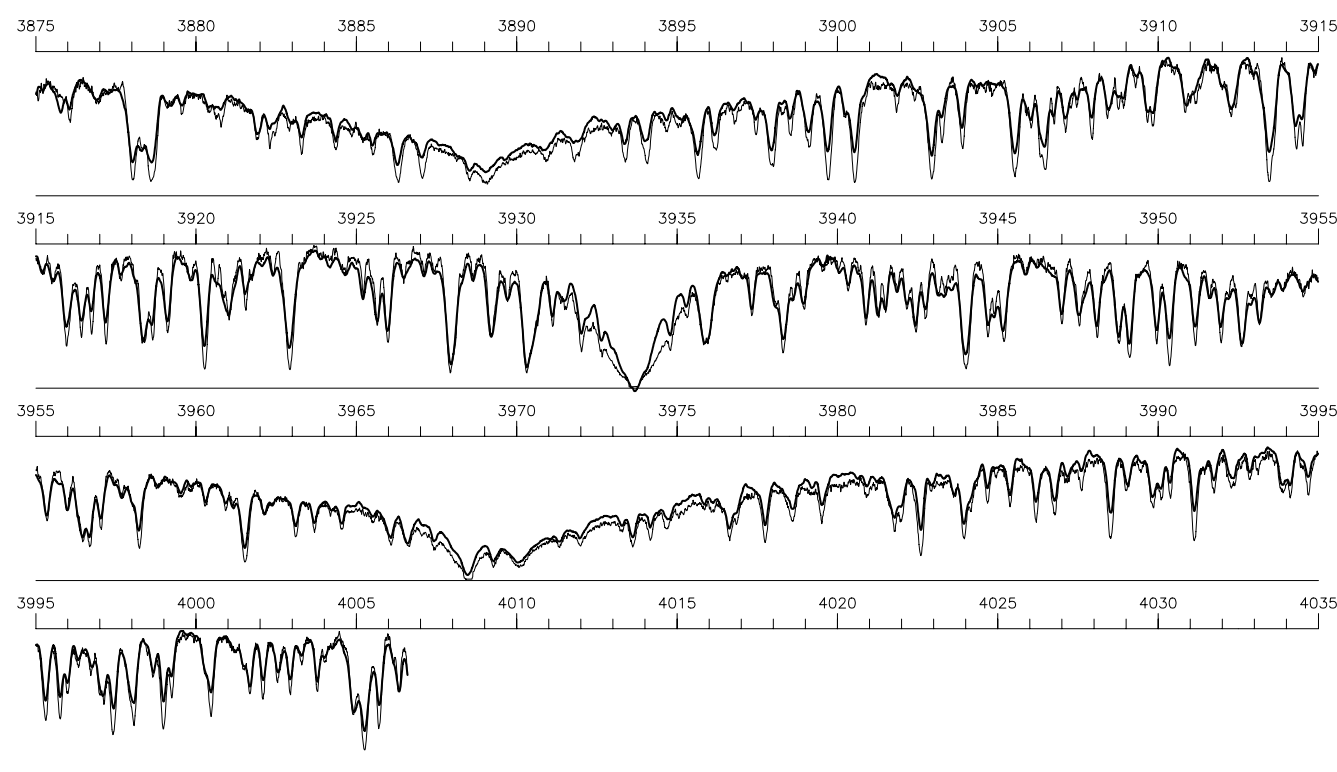

Figure 5. Comparison between the spectra of $o$ Leo B (thick line) and the Am star 32 Aqr (thin line).

\section{Chemical abnormalities of the G0 giant}

The primary seems to share all the general characteristics of Am dwarfs, including a deficiency of $\mathrm{Ca}$ and Sc (see Fig. 7). 
(4420

(460)

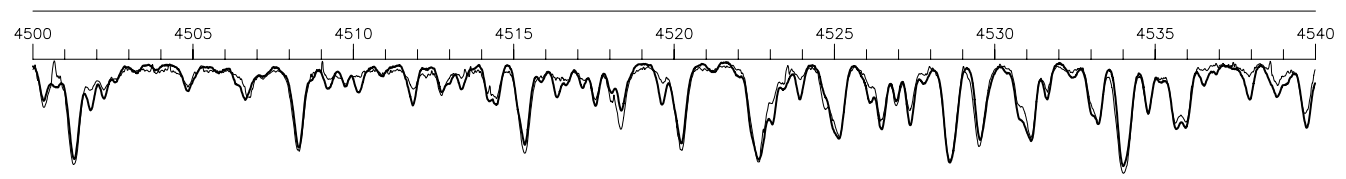

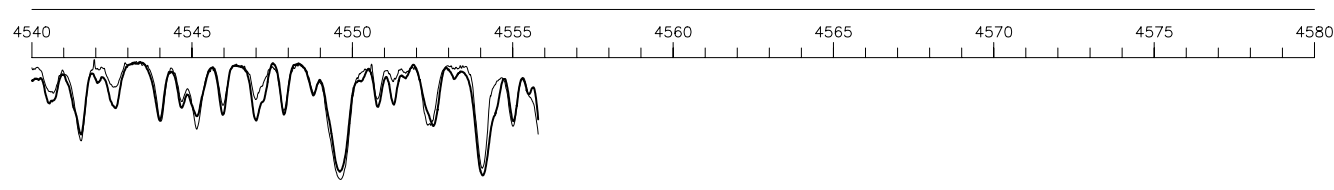

Figure 6. Comparison between the spectra of o Leo A (thick line) and HR 8084, a classical Cepheid (thin line).

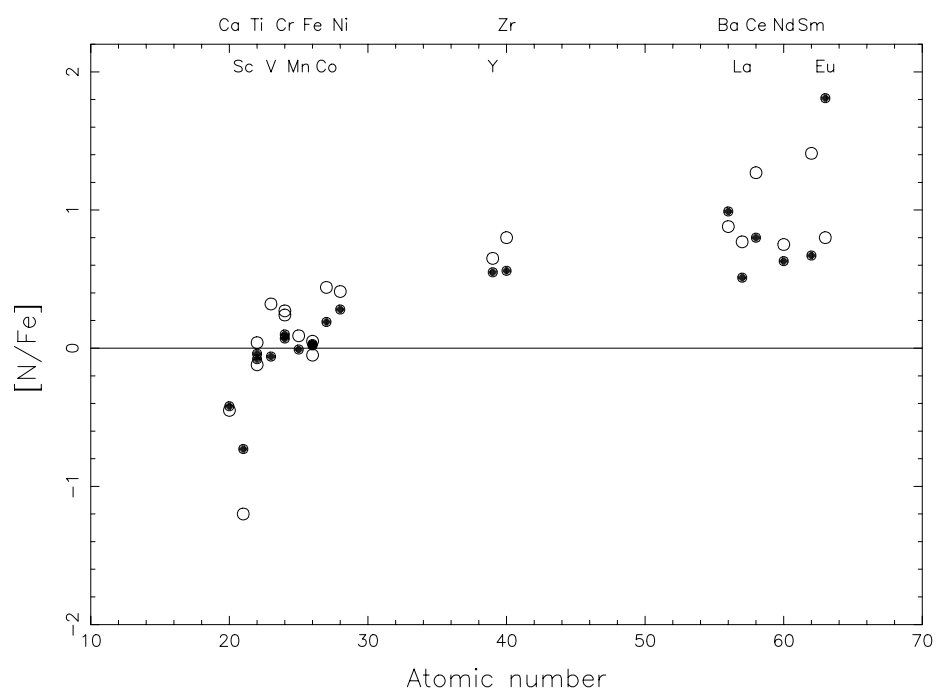

Figure 7. Chemical abundances (relative to Fe) in o Leo A (dots) and an "average" classical Am star (circles).

\section{Evolution of the $o$ Leo binary}

In Fig. 8 we match the HR-diagram position of $o$ Leo with evolutionary tracks drawn for the corresponding masses. The dotted lines represent the boundaries of the pulsational instability strip. In Fig. 9 the isochrone for $1.02 \times 10^{9}$ years (Pols et al. 1998) has been fitted to the points representing the component stars. 


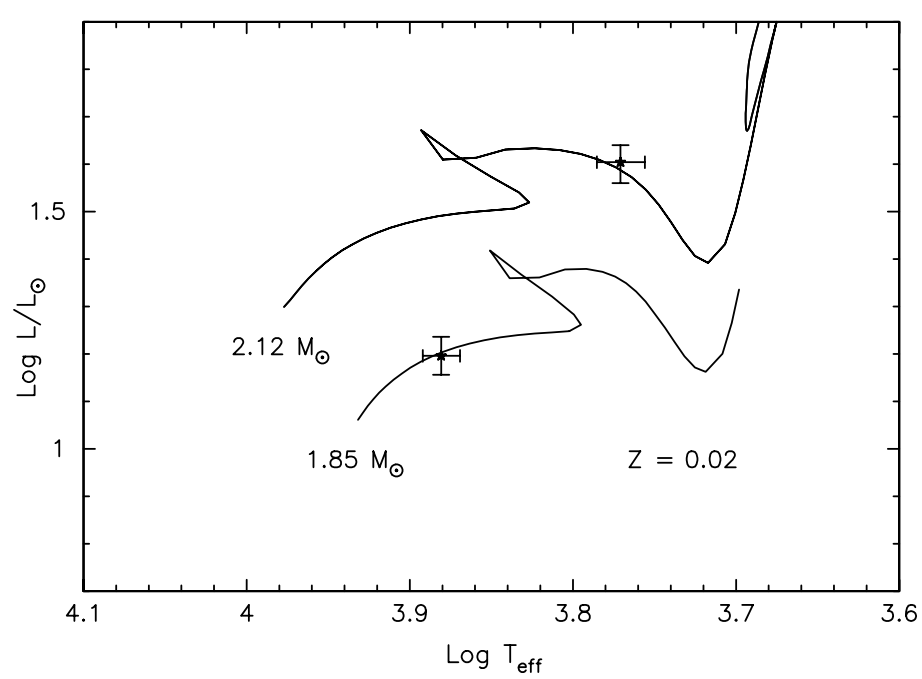

Figure 8. Evolutionary tracks (Pols et al. 1998) for the component stars. Both models have solar compositions.

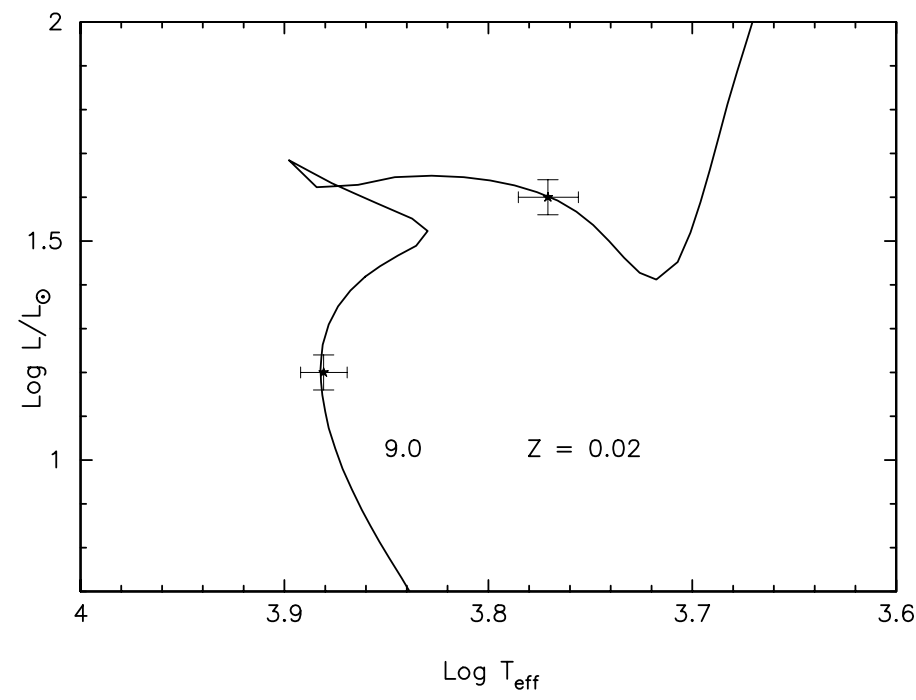

Figure 9. Isochrone for $1.02 \times 10^{9}$ years (Pols et al. 1998), fitted to the points representing the component stars

\section{7. o Leo: freak, or merely rare?}

The secondary is rotating at $8 \mathrm{~km} \mathrm{~s}^{-1}$, probably synchronously. There does not appear to be any chromospheric activity. From the present dimensions of the components, mass exchange is unlikely to have taken place in the past, but it could be expected to play a significant role in the relatively near future.

The primary is the more abnormal of the two components. It seems to be similar to a hot star in the cool-star domain:

- It has near-solar temperature but displays all the spectral characteristics of A-star 
metallicity, yet according to theory the diffusion timescale becomes too long below $T_{\text {eff }} \sim$ $6300 \mathrm{~K}$.

- Its high microturbulence $\left(4.3 \mathrm{~km} \mathrm{~s}^{-1}\right)$ is characteristic of Am stars, not cool giants.

- It is rotating at $11 \mathrm{~km} \mathrm{~s}^{-1}$, about $50 \%$ too slowly to be synchronous. Possibly it was rotating synchronously while on the MS, but now its radius is expanding too quickly for synchronous rotation to be maintained.

This curious object raises a shoal of questions:

- Is the giant still in a process of transition from dwarf to giant, such that it has neither shed its dwarf characteristics nor yet acquired the giant ones?

- Its chemical abundances imitate the pattern of Am stars, though they are not as extreme as some Am stars. Was it necessarily a former Am dwarf?

- Can tidal forces limit the onset of the high rotation that is normal in late-F and early-G giants as they cross the Hertzsprung Gap?

- How rare is it to observe a yellow giant in this phase? The phase of evolution in question is extremely rapid. o Leo may happen to represent a normal evolutionary state of an Am binary. Its apparent rarity could therefore simply reflect the rapidity of its present evolutionary phase.

\subsection{Other stars thought to be evolving Am dwarfs}

If $o$ Leo A has an evolutionary history that is common to Am stars in general, we would expect to identify other stars that have similar properties. Two groups of stars into which Am dwarfs evolve are (A) the $\delta$ Del stars and (B) the AmFm giants. But there are no clear precursors, and in fact $o$ Leo A does not fit either group decisively.

A. Stars like $\delta$ Del are $1000 \mathrm{~K}$ or more hotter than $o$ Leo A. They exhibit Rare Earth overabundances similar to those found in o Leo A, but little (if any) underabundances of $\mathrm{Ca}$ and $S c$. They also pulsate. There has not yet been a firm decision as to whether or not $o$ Leo pulsates, but if it does so then the amplitude is low.

B. The AmFm giants show the same traits in chemical composition as the $\delta$ Del stars, but other general characteristics (stellar rotation, mass, binary membership) set them apart from Am stars.

The relationships among the classical Am, the $\delta$ Del and the AmFm giant stars are not fully understood. The results for $o$ Leo confuse rather than clarify the situation. One key unknown is: Which conditions are necessary for pulsations to occur, and which are merely sufficient? There must be stars in the critical domain which have never been Am stars, and there are Am stars like $o$ Leo A which recently passed through the instability strip and which may not exhibit low-amplitude pulsations.

Possible "intrinsic" explanations include contamination during stellar formation, or a complex magnetic field configuration that leaves no net observable component or Ap characteristics. Both are hard to prove.

A possible "extrinsic" explanation requires accreted metal-rich material from a companion star, as is thought to be the cause of the "Ba" phenomenon. The link, if any, between Am and $\mathrm{Ba}$ stars is suggestive, but may only be coincidental. If the chemicalabundance anomalies in $o$ Leo A are explained in this way, then either heavy-element synthesis has occurred internally, or there is a third, now very evolved, body in the system. The evidence against these possibilities is fairly strong: (i) o Leo A is not evolved enough for the production of $s$-process elements, (ii) its clear overabundance of Eu cannot be explained by that route, and (iii) its RV period is stable and offers no hint of a drift. 


\section{Temporary conclusions}

Omicron Leo seems to be in a class of its own, and the primary in particular shows many unusual and unexpected properties. However, they are more in the nature of extremes than abnormalities, and it seems probable that rational explanations can be given for all the peculiarities discussed above. The giant only seems rare because it happens to be in an evolutionary phase that is extremely rapid, and its present uniqueness is due entirely to that rarity. Rather than necessitating new theories, its analysis sets valuable new constraints on existing ones (such as the diffusion theory). Its rarity does, however, set it apart as an extremely valuable object for further study.

This study emphasizes the potential of high resolution for bright-star research. o Leo is magnitude 3.5, yet it took over 100 years of RV observations before any peculiarities in the spectra of either component were brought to light.

It also reminds us of the importance of binary-star spectroscopy for studies of singlestar evolution.

\section{Acknowledgements}

I am very grateful to the DAO and to the Symposium organizers for grants that enabled me to attend this meeting.

\section{References}

Hadrava, P. 1995, A\& AS 114, 393

Ilijić, S., Hensberge, H. \& Pavlovski, K. 2001, Lecture Notes in Physics 573, 269

Pols, O.R., Schröder, K.-P., Hurley, J.R., Tout, C.A. \& Eggleton, P.P. 1998, MNRAS 298, 525 